\title{
ANÁLISE DE COMPOSTOS SULFURADOS EM EFLUENTES GASOSOS DE REFINARIA DE PETRÓLEO
}

Júlio Carlos Afonso*

Departamento de Química Analítica, Instituto de Química, Universidade Federal do Rio de Janeiro, Av. Athos da Silveira Ramos, 149, 21941-909 Rio de Janeiro - RJ, Brasil

Kátia da Silva Pereira ${ }^{*}$

Petróleo Brasileiro S. A., Refinaria Duque de Caxias, Av. Washington Luis, km 113,7, 25213-005 Duque de Caxias - RJ, Brasil

Recebido em 4/5/09; aceito em 26/10/09; publicado na web em 12/3/10

\begin{abstract}
ANALYSIS OF SULFUR COMPOUNDS IN PETROLEUM REFINERY GASEOUS EFFLUENTS. This work discusses an analytical procedure for analysis of sulfur compounds in treated petroleum refinery gaseous effluents using a sulfur chemiluminescence detector with dual plasma burner (SCD-DP). Calibration was accomplished by using standards and gaseous streams of known concentration of sulfur compounds. The response factors agree with the calibration table of ASTM standard D 5504 (2008). The detection range for sulfur compounds is in $\mu \mathrm{g} \mathrm{m}^{-3}$. The analytical procedure allowed the construction of a chromatographic chart of sulfur compounds present in several refinery gaseous effluents. $\mathrm{SO}_{2}$ was the most difficult compound to be determined because of its high reactivity.
\end{abstract}

Keywords: sulfur compounds; chemiluminescence; gaseous effluents.

\section{INTRODUÇÃO}

Atualmente, as decisões sobre a viabilidade de um empreendimento na indústria de refino de petróleo incluem o componente ambiental, pois é um item fundamental na análise do processo e de sua rentabilidade. Desse modo, as refinarias investem na minimização da geração de resíduos, adotando métodos como a utilização de tecnologias de processamento de poluentes reativos como compostos de enxofre. Os processos de refino de petróleo são responsáveis pela emissão de dióxido de enxofre $\left(\mathrm{SO}_{2}\right)$ ou de sulfeto de hidrogênio $\left(\mathrm{H}_{2} \mathrm{~S}\right) .{ }^{1}$

$\mathrm{O} \mathrm{SO}_{2}$ tem potenciais efeitos danosos ao organismo, pois concentrações de $1,2 \mathrm{mg} \mathrm{m}^{-3} \mathrm{em}$ ambientes fechados provocam constrições no sistema respiratório de pessoas saudáveis. ${ }^{2}$ Concentrações elevadas de $\mathrm{SO}_{2}$ em certas situações provocam deposição ácida e smog industrial. ${ }^{3,4} \mathrm{Já}$ o $\mathrm{H}_{2} \mathrm{~S}$ leva à morte imediata em concentrações a partir de $1000 \mathrm{mg} \mathrm{m}^{-3}$ no ambiente. ${ }^{2}$ Além disso, é oxidado a $\mathrm{SO}_{2}$ na atmosfera num tempo médio de $24 \mathrm{~h} .^{5}$

A unidade de recuperação de enxofre (URE) é a tecnologia para reduzir o impacto ambiental devido aos compostos sulfurados. ${ }^{6-8} \mathrm{~A}$ carga da URE, rica especialmente em $\mathrm{H}_{2} \mathrm{~S},{ }^{6}$ é a mistura de gases provenientes principalmente das unidades de pré-tratamento de correntes dos processos de craqueamento catalítico em leito fluidizado (Fluid Catalytic Cracking - FCC); hidrotratamento de querosene e óleo diesel; dessulfurização de óleos parafínicos e lubrificantes, e tratamento de águas ácidas. ${ }^{4}$ Todas essas correntes gasosas têm caráter ácido. ${ }^{7,8} \mathrm{Os}$ principais compostos sulfurados responsáveis pela característica ácida das mesmas são $\mathrm{H}_{2} \mathrm{~S}$, sulfeto de carbonila (COS) e tióis leves como metanotiol, etanotiol, propanotióis e butanotióis. A passagem em torres de absorção com aminas reduz em mais de $95 \%$ a concentração desses sulfurados, sendo considerado tratamento preliminar de gases ácidos em refinaria de petróleo. ${ }^{8}$ A dietanolamina (DEA) é amplamente utilizada para esse fim devido à possibilidade de ser regenerada mediante aquecimento a $125^{\circ} \mathrm{C}$; os compostos sulfurados são dessorvidos. ${ }^{9} \mathrm{O}$ emprego da DEA também se justifica porque ela reage seletivamente com $\mathrm{H}_{2} \mathrm{~S}$ com maior rendimento que a monoetanolamina (MEA),

*e-mail: julio@iq.ufrj.br

"Atualmente no Centro de Pesquisas Leopoldo Miguez de Mello

(CENPES/PETROBRAS) formando produtos estáveis à temperatura ambiente; a DEA reage reversivelmente com COS, enquanto que a MEA forma compostos não regeneráveis; a DEA absorve tióis à temperatura ambiente. ${ }^{10} \mathrm{O}$ gás efluente após a regeneração é enviado à URE.

As correntes gasosas ácidas apresentam alta corrosividade, sendo danosas às estruturas de transporte e de processos industriais, e são nocivas aos catalisadores usados em processos de reforma, FCC e hidrocraqueamento, mesmo em baixas concentrações, devido ao envenenamento de seus sítios ativos. ${ }^{11}$ Por isso, o monitoramento das concentrações de compostos sulfurados residuais nos gases ácidos após tratamento em torres de absorção é fundamental, tanto para verificar a eficácia dos processos de remoção desses compostos, como também para prevenir danos aos principais processos de refino do petróleo. . $^{3,7,7,8,11}$

O processo para recuperação de enxofre a partir dos compostos removidos dos gases ácidos é baseado na oxidação do $\mathrm{H}_{2} \mathrm{~S}$, de acordo com os princípios da reação de Claus. Ele foi patenteado em $1883 \mathrm{em}$ Londres pelo químico Carl F. Claus (1835-1899). ${ }^{12}$ Nesse processo, $\mathrm{o}_{2} \mathrm{~S}$ é convertido em enxofre elementar, substância não agressiva do ponto de vista ambiental, segundo as reações:

$$
\begin{aligned}
& 2 \mathrm{H}_{2} \mathrm{~S}+3 \mathrm{O}_{2} \rightleftarrows 2 \mathrm{SO}_{2}+2 \mathrm{H}_{2} \mathrm{O} \\
& 2 \mathrm{H}_{2} \mathrm{~S}+\mathrm{SO}_{2} \rightleftarrows 3 \mathrm{~S}_{8}+2 \mathrm{H}_{2} \mathrm{O}
\end{aligned}
$$

A recuperação de enxofre se situa entre 95 e $99 \%$ m/m.,12 O enxofre recuperado é vendido para indústrias de fertilizantes, cosméticos, medicamentos, papel e celulose. ${ }^{8}$

\section{Cromatografia e detecção de compostos sulfurados por quimiluminescência}

Quimiluminescência é definida como emissão de luz pelo resultado de uma reação química que fornece espécie excitada eletronicamente, transferindo energia para outra espécie. ${ }^{13,14}$ Os métodos de quimiluminescência são altamente sensíveis porque níveis baixos de luz são facilmente monitorados. ${ }^{14} \mathrm{~A}$ conversão dos compostos contendo enxofre para suas respectivas espécies quimiluminescentes e sua detecção tem larga aplicação em química analítica, sendo um dos destaques a associação com a cromatografia gasosa. 
Os métodos de quimiluminescência para a determinação de componentes em gases originaram-se a partir da necessidade de se determinar poluentes atmosféricos combinando alta seletividade e sensibilidade. O desenvolvimento na aplicação da detecção quimiluminescente ocorreu entre as décadas de 1970 e 1990 e desde então vem crescendo a comercialização de detectores baseados na quimiluminescência induzida por ozônio $\left(\mathrm{O}_{3}\right) \cdot{ }^{13,14}$

Dentre os sistemas de detecção quimiluminescentes mais usados com cromatografia gasosa para a determinação de compostos sulfurados nos últimos anos destaca-se o detector quimiluminescente de enxofre com queimador de plasma duplo: SCD-DP (sulfur chemiluminescence detection with dual plasma). Embora numerosas reações quimiluminescentes ocorram entre ozônio e espécies presentes no analito, o sistema SCD-DP possui filtro óptico de UV, localizado entre a câmara de reação e a fotomultiplicadora, que transmite a luz emitida pela reação de ozonização seletivamente para a banda 225$250 \mathrm{~nm}$ para detecção das espécies quimiluminescentes metaestáveis de enxofre. ${ }^{13} \mathrm{O}$ SCD com queimador de plasma duplo é um sistema relativamente recente ${ }^{15} \mathrm{e}$ ainda pouco explorado no refino de petróleo, sendo importante estudar as possibilidades e limitações desse sistema aplicado na análise de efluentes gasosos..$^{15-17}$

A análise de compostos sulfurados em $\mu \mathrm{g} \mathrm{m}^{-3} \mathrm{e} \mathrm{mg} \mathrm{m}^{-3}$ apresenta como desafios a coleta de amostra e a obtenção de repetitividade da resposta analítica. Assim, os objetivos deste trabalho foram voltados para garantir a representatividade das amostras e a otimização de um sistema GC-SDD-DP para análise de compostos sulfurados em níveis até $\mu \mathrm{g} \mathrm{m}^{-3}$ em correntes gasosas de uma refinaria de petróleo.

\section{PARTE EXPERIMENTAL}

\section{Seleção das correntes gasosas}

Foram consideradas cinco correntes gasosas relativas aos processos de tratamento da carga para FCC, destilação atmosférica de duas plantas (combustíveis e lubrificantes) e saída dos regeneradores da DEA na Refinaria Duque de Caxias (REDUC/Petrobras). Essas correntes se somam a outras, sendo enviadas à URE. O efluente na saída do último condensador de enxofre da URE foi selecionado como a quinta corrente gasosa para este estudo.

\section{Procedimento de amostragem}

Para a coleta de amostras de efluentes com pressão próxima à atmosférica (correntes de saída dos regeneradores da DEA, das unidades de destilação atmosférica e de FCC), foram utilizados sacos de teflon, com capacidade de $500 \mathrm{~mL}$, novos e sob vácuo. $\mathrm{O}$ teflon foi usado no lugar da ampola de vidro devido aos perigos e danos inerentes à quebra deste material, somados à baixa disponibilidade de tempo de estocagem para realização dos ensaios com garantia de concentração de sulfurados com $95 \%$ de confiança (a amostra permanece íntegra no saco de teflon por até $8 \mathrm{~h}^{18}$ ). Para as amostras do efluente na saída do último condensador de enxofre, com pressão ligeiramente negativa, foram usadas bombas de sucção com cobertura de teflon para seus dutos internos e sondas de aspiração de gases com posterior confinamento em sacos de teflon. Esse efluente é caracterizado pela presença simultânea de $\mathrm{H}_{2} \mathrm{~S}, \mathrm{SO}_{2}$ e $\mathrm{H}_{2} \mathrm{O}$. O SO pode reagir com a água segundo a reação 3 e com $\mathrm{H}_{2} \mathrm{~S}$ segundo a reação $2 .{ }^{4} \mathrm{Na}$ coleta é necessário secar o gás imediatamente antes da introdução deste no recipiente de coleta, do contrário ocorre diminuição considerável da concentração de $\mathrm{SO}_{2}$ durante a própria coleta e o transporte para o laboratório. A amostra gasosa é passada por tubo contendo $\mathrm{P}_{4} \mathrm{O}_{10}$ seco, onde ocorre a reação 4 .
$\mathrm{SO}_{2}+\mathrm{H}_{2} \mathrm{O} \rightarrow \mathrm{H}_{2} \mathrm{SO}_{3}$

$\mathrm{P}_{4} \mathrm{O}_{10}+6 \mathrm{H}_{2} \mathrm{O} \rightarrow 4 \mathrm{H}_{3} \mathrm{PO}_{4}$

Os pontos de amostragem selecionados apresentam-se localizados de forma que o gás a ser coletado ( 3 amostras por coleta) se encontra à temperatura ambiente. As coletas foram feitas em dias sem chuva, no período de maior atividade do processo. Os compostos sulfurados presentes nas amostras podem se decompor com o passar do tempo sob ação do calor e da luz. Por isso, foi utilizado um veículo destinado especificamente a essa tarefa a fim de diminuir o tempo entre a coleta e a recepção no laboratório, e ainda frasqueiras opacas com isolamento térmico para acondicionamento do material de amostragem.

\section{Descrição do cromatógrafo}

O cromatógrafo com SCD-DP usado neste trabalho possui coluna cromatográfica em tubo sulfinert ( $2 \mathrm{~m}$ de comprimento e 0,75 $\mathrm{cm}$ de diâmetro) contendo dimetil polissiloxano empacotada com polímeros inertes (100/200 mesh) como fase estacionária. Devido à reatividade dos compostos sulfurados, em especial do $\mathrm{SO}_{2}$, o uso dessa coluna é adequado para limites de quantificação até níveis até $\mu \mathrm{g} \mathrm{m} \mathrm{m}^{-3} \mathrm{com}$ garantia de qualidade metrológica. ${ }^{17} \mathrm{O}$ sistema de injeção da amostra é constituído por um loop de $1 \mathrm{~mL}$ em tubo sulfinert a 30 ${ }^{\circ} \mathrm{C}$ e 6 válvulas de alumínio (valco) aquecidas a $150{ }^{\circ} \mathrm{C}$. A inércia química passa por avaliações periódicas quanto ao nível de sinal gerado pela injeção de padrão de $\mathrm{SO}_{2}$ (composto sulfurado mais reativo). $\mathrm{O}$ esquema detalhado da unidade de análise encontra-se na literatura. ${ }^{18}$ As condições de análise foram: temperatura do sistema de injeção, $150{ }^{\circ} \mathrm{C}$; temperatura inicial do forno, $45^{\circ} \mathrm{C}$, por $1 \mathrm{~min}$; velocidade de aquecimento, $10^{\circ} \mathrm{C} \mathrm{min}^{-1}$; temperatura final, $195{ }^{\circ} \mathrm{C}$, por $24 \mathrm{~min}$; gás carreador, hélio $\left(11,3 \mathrm{~mL} \mathrm{~min}^{-1}\right)$; temperatura do detector, $800^{\circ} \mathrm{C}$; pressão do detector, $400-410$ torr.

\section{Descrição do sistema de detecção cromatográfico}

Foi utilizado o sistema SCD-DP cuja configuração, quando comparada às outras técnicas de detecção seletiva dos compostos sulfurados, apresenta elevada estabilidade e capacidade de quantificar a resposta de sulfurados em níveis de até $\mu \mathrm{g} \mathrm{m}^{-3}$. Suas partes constituintes são: (a) queimador plasma de chama dupla; (b) tubo cerâmico; (c) câmara de reação; (d) fotomultiplicadora; (e) filtro óptico do detector; (f) gerador de $\mathrm{O}_{3}$. O tubo de cerâmica, que possui função catalítica, apresenta formato longo o que contribui para a estabilidade no desempenho do detector. ${ }^{15}$

$\mathrm{O}$ efluente da coluna cromatográfica reage com $\mathrm{O}_{2}$ na base do queimador e sofre oxidação antes da zona central da chama, rica em misturas $\mathrm{O}_{2} / \mathrm{H}_{2}$. No queimador as espécies quimiluminescentes geradas através da oxidação no plasma, seguida de pirólise redutora no tubo cerâmico, são coletadas e removidas da chama, usando-se uma sonda de cerâmica de alta pureza em linha com uma bomba de vácuo e, finalmente, é enviada para a câmara de reação de ozonização. $\mathrm{O}$ gerador de $\mathrm{O}_{3}$ produz excesso deste através da descarga de alta voltagem na corrente de ar. $\mathrm{O}_{3}$ é responsável pela reação de formação de $\mathrm{SO}_{2}$ eletronicamente excitado, que relaxa com emissão de luz na região azul e ultravioleta do espectro. A luz produzida na reação é detectada com tubo fotomultiplicador sensível ao azul e o sinal é amplificado pelo sistema de dados, com produção de pico cromatográfico. Os compostos que não contêm enxofre são convertidos a espécies $\mathrm{CO}_{2}$ e $\mathrm{H}_{2} \mathrm{O}$ que não reagem com $\mathrm{O}_{3}$ para produzir quimiluminescência perceptível na região espectral usada para detecção de enxofre. ${ }^{13}$ As reações de con- 
versão de compostos sulfurados em espécies quimiluminescentes são descritas na literatura. ${ }^{19} \mathrm{~A}$ arquitetura desse detector (Figura 1) favorece a sensibilidade requerida de detecção, pois a câmara de reação está localizada de modo que a reação entre compostos quimiluminescentes e $\mathrm{O}_{3}$ ocorra diretamente à frente do tubo da fotomultiplicadora, favorecendo a detecção da intensidade da emissão quimiluminescente. ${ }^{13}$

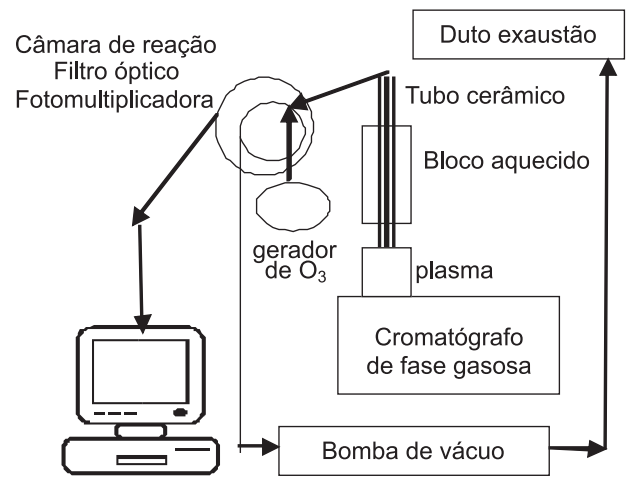

Figura 1. Representação esquemática de sistema cromatográfico com detector quimiluminescente

O detector trabalha sob vácuo (bomba de alto vácuo Welch refrigerado a ar com pistão seco), que intensifica a quimiluminescência, uma vez que ela é inversamente proporcional à pressão na câmara de reação com $\mathrm{O}_{3}$, mantém a pressão de vapor d'água abaixo do ponto de orvalho na câmara de reação com $\mathrm{O}_{3}$ e previne a condensação que pode ocorrer na câmara ou nas linhas de transferência das correntes gasosas. ${ }^{13} \mathrm{O}$ vácuo ainda transfere os gases da chama para a câmara de reação, do $\mathrm{O}_{3}$ do gerador para a câmara de reação e das correntes gasosas da câmara de reação para trap químico (solução aquosa de $\mathrm{KI}$, que elimina o excesso de $\mathrm{O}_{3}$ ).

\section{Métodos de identificação de compostos sulfurados}

A metodologia adotada, para a identificação dos compostos sulfurados de interesse na área de refino de petróleo, é a determinação de seus tempos de retenção, utilizando três técnicas de trabalho distintas e complementares.

\section{Uso de material de referência certificado (MRC)}

A identificação dos compostos sulfurados ocorre com o uso de MRC, durante os testes de repetitividade de cada composto sulfurado, até o nível de $\mu \mathrm{g} \mathrm{m}^{-3}$, gerado através de tubos de permeação ( $\mathrm{H}_{2} \mathrm{~S}$; COS; $\mathrm{SO}_{2}$; metanotiol (MESH); dimetilsulfeto (DMS); dimetildissulfeto (DMDS)). O equipamento gerador de referência gasosa utilizado foi o dynacalibrator, modelo 230 (VICI Metronics).

\section{Uso de amostra de referência}

A identificação dos compostos sulfurados ocorre mediante o emprego de amostra de referência oriunda de processamento de gás natural. Este sofre odorização com mistura de tióis de composição conhecida. Os compostos 2-propanotiol, 2-(2-metil)propanotiol e 1-propanotiol apresentam-se numa proporção de 77, 15 e 8\% vol., respectivamente.

\section{Uso de tabelas de índices de retenção Kovats}

Listas de índices de retenção de Kovats para 21 compostos sulfurados foram usadas em associação com as informações de densidades e pontos de ebulição dos mesmos. ${ }^{19-21}$

\section{Método de ensaio e melhorias realizadas no sistema cromatográfico}

A técnica analítica para o controle de sulfurados na faixa de $\mu \mathrm{g} \mathrm{m}^{-3}$ foi baseada no método ASTM D 5504 (método padrão para determinação de compostos sulfurados em gás natural e gases combustíveis por cromatografia gasosa e quimiluminescência). ${ }^{19}$

As características do SCD-DP relevantes para o trabalho de otimização de sua resposta são a seletividade para compostos sulfurados, sensibilidade da detecção, equimolaridade para diferentes compostos sulfurados e linearidade na faixa de trabalho.

Para minimizar efeitos de matriz de hidrocarbonetos foi selecionada uma chama rica em hidrogênio $\left(\mathrm{H}_{2} / \mathrm{ar} 2: 1 \mathrm{v} / \mathrm{v}\right)$ no queimador, que favorece a combustão completa da amostra, promovendo geração máxima de espécies quimiluminescentes. O SCD-DP desempenha papel fundamental neste processo de queima, pois apresenta duas chamas em sequência que contribuem para que a detecção seja mais estável quando comparada ao do SCD simples..$^{15,16}$

A fim de adaptar o método ASTM D 5504 para análise de gases efluentes de refinaria de petróleo, na faixa de 0,010 a $1000 \mathrm{mg} \mathrm{m}^{-3}$, incluindo o $\mathrm{SO}_{2}$, foi feito um estudo de otimização da resposta analítica, com o objetivo de obter resultados com precisão e exatidão buscadas, para a faixa de trabalho requerida, evitando-se desvios de linearidade e nível de ruído interferente, além de promover condições para superar o limite para concentração mínima detectável. Os parâmetros otimizados são: (a) avaliação da inércia química dos dutos do sistema de injeção e coluna cromatográfica através da repetitividade da resposta do $\mathrm{SO}_{2}$ e determinação do limite de quantificação; (b) obtenção da melhor resolução da coluna para $\mathrm{H}_{2} \mathrm{~S}$ e $\mathrm{SO}_{2}$ no menor tempo de corrida analítica; (c) determinação do tempo de estabilização na geração de $\mathrm{H}_{2} \mathrm{~S}$ e $\mathrm{SO}_{2}$ (MRC) nas condições de passivação do sistema cromatográfico.

\section{Calibração do SCD-DP}

A calibração requer a quantificação por classe de compostos sulfurados, pois a resposta do detector é teoricamente equimolar para cada uma delas. A quantificação dos diferentes compostos sulfurados é determinada com precisão similar padronizando-se a concentração de um composto sulfurado de cada família de interesse: tióis, monossulfetos e dissulfetos. Neste trabalho foram também considerados os seguintes compostos: $\mathrm{H}_{2} \mathrm{~S}, \mathrm{COS}$ e $\mathrm{SO}_{2}$. Como a resposta do SCD é proporcional ao mol de enxofre, todos os compostos monossulfurados terão aproximadamente o mesmo fator de resposta para $\mu \mathrm{g} \mathrm{m}^{-3}$ de enxofre.

Neste trabalho foram selecionados dois tipos de MRC para a calibração da resposta do SCD-DP: tubos de permeação gasosa (também usados na identificação dos compostos sulfurados) e, misturas de referência gasosa industriais (White Martins). Na geração do material de referência foi usado o equipamento dynacalibrator segundo os ensaios ASTM D 3609, ASTM D 4298 e ASTM E 94 (capacidade de vazão de $175 \mathrm{~mL} \mathrm{~min}^{-1}$ no fluxo de arraste e $5 \mathrm{~L} \mathrm{~min}^{-1}$ no fluxo total, com câmara de diluição em pirex, calibrada a $30^{\circ} \mathrm{C}$, válvula micrométrica frontal para ajuste de gás de arraste e rotâmetros de precisão, bomba de sucção, controle de temperatura). ${ }^{22-24}$ As misturas de referência, pressurizadas em cilindro de alumínio de $4 \mathrm{~L}$ (volume interno), foram: metanotiol $\left(62,53 \pm 3,13 \mathrm{mg} \mathrm{m}^{-3}\right)$ em butano, $\mathrm{SO}_{2}\left(109 \pm 3 \mathrm{mg} \mathrm{m}^{-3}\right)$ e $\mathrm{SO}_{2}\left(498 \pm 6 \mathrm{mg} \mathrm{m}^{-3}\right)$, ambos em propeno. O MRC em cilindro de alumínio ainda é muito usado em calibração de instrumentos de linha de gases de refinarias. ${ }^{25,26}$

A concentração da mistura gasosa de referência, gerada a partir de tubos de permeação MRC (nível de 95\% de confiança), é determinada pela perda de massa pela passagem de fluxo contro- 
lado de gás e de acordo com os seguintes parâmetros de medição, conforme a norma ASTM D 4298: ${ }^{23}$ taxa da vazão de ar para diluição; temperatura na câmara de permeação; taxa de permeação do tubo, cujo valor nominal é informado no tubo de permeação; temperatura e pressão do ar ambiente usado para promover a permeação; vazão final da geração do MRC medido na saída do cromatógrafo (fluxímetro digital). Esta forma de trabalho apresenta vantagem de estabilidade da concentração e vida útil longa do material de referência de calibração (até 1 ano), pois a calibração da taxa de permeação do tubo é válida até $90 \%$ de o líquido sofrer permeação. ${ }^{22} \mathrm{~A}$ verificação da resposta do detector foi feita usando gás natural (80-92\% vol. metano, $5-15 \%$ vol. de etano, $1-3 \%$ vol. de propano, $\leq 1 \%$ de butanos e homólogos superiores, 1-1,5 $\%$ vol. de inertes) odorizado em linha por bomba dosadora que injeta uma mistura de três compostos sulfurados: 2 propanotiol, 2-(2-metil)propanotiol e 1-propanotiol. As concentrações, a partir de $2 \mathrm{~m}$ da bomba dosadora, foram calculadas a partir do volume injetado de odorizantes e do volume do gás movimentado através do gasoduto a partir da vazão, pressão e temperatura do mesmo. A comparação entre os valores obtidos pelo sistema da bomba dosadora e da análise da amostra (usando fatores de correção para SCD-DP obtidos de MRC a partir de tubos de permeação) mostrou excelente fator de recuperação para os três compostos sulfurados na matriz de hidrocarbonetos.

Na calibração, são importantes os seguintes parâmetros: equimolaridade, ou seja, a resposta do SCD para o mesmo número de átomos de enxofre é a mesma, independente da estrutura molecular do analito ou da complexidade da matriz da amostra; linearidade, definida como a razão entre a maior e a menor concentração da amostra onde a resposta do detector é linear - de acordo com a norma ASTM D 5504 a faixa de linearidade é de $10^{1}$ a $10^{6} \mu^{-3} \mathrm{~m}^{-3}$ de enxofre; ${ }^{19}$ sensibilidade, determinada a partir da área do enxofre correspondente à massa do elemento na amostra no limite de quantificação. ${ }^{19,24}$ Para a determinação da sensibilidade do SCD-DP foi selecionado o cálculo tradicional que considera o sinal de resposta do SCD por unidade de massa do composto sulfurado. Este método é o primeiro dos três recomendados na norma ASTM E 594, adaptado para SCD: $:^{24}$

$\mathrm{S}\left(\mu \mathrm{g} \mathrm{S} \mathrm{s}^{-1}\right)=60 \mathrm{~m} \mathrm{~A}^{-1}$

onde: $\mathrm{S}=$ sensibilidade; $\mathrm{m}=\mu \mathrm{g} \mathrm{S}$ do $\mathrm{MRC}$ gerado por tubo de permeação; $\mathrm{A}=$ sinal de resposta com contador em minutos; $60=$ conversão do contador de área de min para s.

\section{RESULTADOS E DISCUSSÃO}

\section{Validação da resposta do SCD-DP}

O sumário da validação da resposta do SCD-DP, apresentado na Tabela 1, compara as características de desempenho dos detectores, a partir dos dados obtidos, com os dados de literatura. A calibração do SCD-DP com tubos de permeação apresentou fatores de resposta correlacionáveis àqueles tabelados pelo método ASTM D 5504-01. ${ }^{19}$

Tabela 1. Resumo das características de desempenho do SCD de plasma duplo

\begin{tabular}{lcc}
\hline Característica & $\begin{array}{c}\text { Teoria } \\
\text { ASTM D5504 }{ }^{19}\end{array}$ & $\begin{array}{c}\text { Prática } \\
\text { SCD de plasma duplo }\end{array}$ \\
\hline Sensibilidade $\left(\mu \mathrm{g} \mathrm{S} \mathrm{s}^{-1}\right)$ & $<5$ & $3 \mathrm{a} 4$ \\
Seletividade $(\mathrm{S} / \mathrm{C})$ & $>10^{6}$ & até 50 vezes $>10^{6}$ \\
Fc e equimolaridade & $\operatorname{sim}$ & $\operatorname{sim}$ \\
Linearidade (exceto $\left.\mathrm{SO}_{2}\right)$ & $10^{5}$ & $10^{4}$ \\
\hline
\end{tabular}

\section{Fator de correção teórico e equimolaridade}

A característica de resposta equimolar baseia-se na etapa de combustão onde ocorre a conversão de todos os compostos de enxofre a espécies quimiluminescentes que contêm este elemento. A Figura 2 indica a similaridade entre sulfetos (representado pelo dimetilsulfeto) e tióis (representado pelo metanotiol); e mostra que a resposta do $\mathrm{SO}_{2}$ apresenta equimolaridade para tióis e sulfetos com mesmo número de átomos de $\mathrm{S}$ que venham a ser usados como MRC na calibração por tubos de permeação.

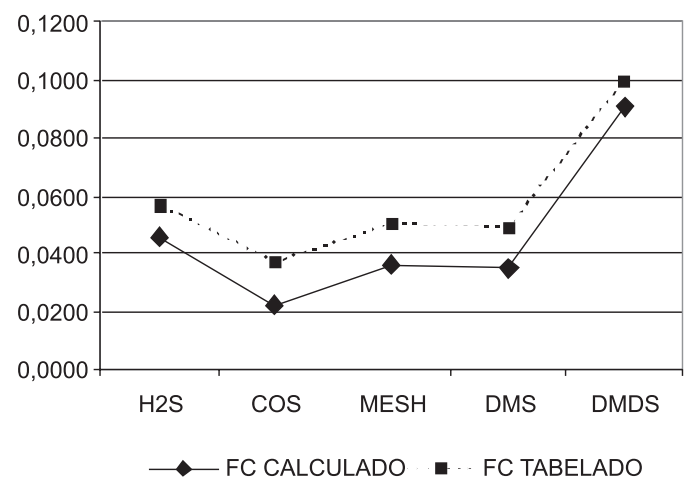

Figura 2. Gráfico de comparação de fatores de correção calculados e tabelados (dados teóricos retirados da Tabela 4 da norma ASTM D 5504 ${ }^{19}$ )

Assim, um material de referência constituído por um composto contendo um átomo de enxofre (exceto $\mathrm{H}_{2} \mathrm{~S}$ e COS) pode ser usado para calibração da resposta do detector para a análise quantitativa de quaisquer outros sulfetos e tióis, mesmo para compostos sulfurados não conhecidos. Para os compostos $\mathrm{H}_{2} \mathrm{~S}$ e COS, onde há um desvio em relação ao fator de resposta dos demais monossulfurados (Figura 2), recomenda-se que se faça a determinação dos fatores de calibração através de calibração específica.

Os fatores calculados apresentaram-se menores que os tabelados e, por consequência, as áreas de resposta do SCD de plasma duplo foram maiores, indicando que o detector se encontra otimizado para limites de quantificação na faixa de $\mu \mathrm{g} \mathrm{m}^{-3}$.

\section{Limite de quantificação (LQ)}

O SCD consegue detectar concentrações de compostos sulfurados em nível de $\mu \mathrm{g} \mathrm{m}^{-3}$. Segundo a norma ASTM D 5504, o limite de detecção é de $10 \mu \mathrm{g} \mathrm{m}^{-3}$ de S. A literatura indica que os limites de detecção mais baixos relatados eram da ordem de $50 \mu \mathrm{g} \mathrm{m}^{-3} \cdot{ }^{13,19}$

Neste trabalho foram calculados os limites de quantificação dos MRC gerados por tubos de permeação (Tabela 2) para cada composto sulfurado.

Tabela 2. Sumário do limite de quantificação dos sulfurados de referência

\begin{tabular}{lcc}
\hline Composto sulfurado & $\begin{array}{c}\mathrm{LQ} \pm \text { desvio padrão } \\
\left(\mu \mathrm{g} \mathrm{m}^{-3}\right)\end{array}$ & $\begin{array}{c}\text { LQ considerado } \\
\left(\mu \mathrm{g} \mathrm{m}^{-3}\right)\end{array}$ \\
\hline $\mathrm{H}_{2} \mathrm{~S}$ & $7 \pm 1$ & 8 \\
$\mathrm{COS}$ & $5 \pm 1$ & 6 \\
$\mathrm{MESH}$ & $10 \pm 3$ & 13 \\
$\mathrm{DMS}$ & $10 \pm 3$ & 13 \\
$\mathrm{SO}_{2}$ & $86 \pm 18$ & 100 \\
$\mathrm{DMDS}$ & $54 \pm 15$ & 70 \\
\hline
\end{tabular}

Como teoricamente o limite de quantificação e o limite de detecção apresentam uma relação de 3:1, este estudo indica um limite de detecção menor para o SCD-DP. Este estudo considera o limite de 
quantificação devido à margem de segurança em trabalhos de ultratraços $\left(\mu \mathrm{g} \mathrm{m}^{-3}\right)$. Outra consideração relevante para registro é a diferença entre $\mathrm{SO}_{2}$ e os demais sulfurados quanto ao nível de concentração do LQ, evidenciando mais uma vez o grau de dificuldade considerável no estudo de determinação do $\mathrm{SO}_{2}$ devido à sua reatividade.

O limite de quantificação é definido como a quantidade do analito que gera resposta três vezes maior que o nível de ruído $;{ }^{27} \mathrm{o}$ limite de detecção é definido como a quantidade do analito que gera resposta duas vezes maior que o nível de ruído pela sensibilidade que, no caso

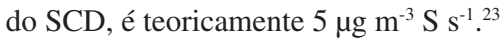

\section{Sensibilidade do SCD}

Segundo a norma ASTM D 5504, a sensibilidade do método deste trabalho é menor que $5 \mu \mathrm{g} \mathrm{m}^{-3} \mathrm{~S} \mathrm{~s}^{-1} .{ }^{19}$ Este método possui elevada sensibilidade sendo um dos motivos o fato da luz emitida no método quimiluminescente ocorrer em câmara isolada, sem interferência de outras fontes de luz, diferente de outros métodos luminescentes. ${ }^{13}$

A Tabela 3 apresenta as sensibilidades calculadas para diversos compostos sulfurados. As áreas listadas são estimadas para os limites de quantificação considerados e registrados nessa Tabela. O cálculo da massa considera a conversão do limite de quantificação para $\mu \mathrm{g}$ $\mathrm{m}^{-3}$ e peso molecular 29 para o ar teórico usado na geração do MRC por tubo de permeação.

Tabela 3. Sumário da determinação da sensibilidade do SCD de plasma duplo

\begin{tabular}{lccc}
\hline $\begin{array}{l}\text { Composto } \\
\text { sulfurado }\end{array}$ & $\begin{array}{c}\text { Área estimada } \\
\text { para LQ }\end{array}$ & $\begin{array}{c}\text { LQ em } \\
\mu \mathrm{g} \mathrm{S}\end{array}$ & $\begin{array}{c}\mathrm{S} \\
\left(\mu \mathrm{g} \mathrm{S} \mathrm{s}^{-1}\right)\end{array}$ \\
\hline $\mathrm{H}_{2} \mathrm{~S}$ & 163610 & 8662 & 3 \\
$\mathrm{COS}$ & 143590 & 6521 & 3 \\
$\mathrm{MESH}$ & 276930 & 16303 & 4 \\
DMS & 221820 & 16276 & 4 \\
DMDS & 526587 & 75940 & 4 \\
\hline
\end{tabular}

A sensibilidade do SCD de plasma duplo está de acordo com aquela estimada pelo ASTM D 5504 e, portanto, este sistema está validado para o item sensibilidade. ${ }^{19}$

\section{Linearidade do SCD e faixa de trabalho}

Os detectores de quimiluminescência apresentam resposta linear correspondente à concentração de enxofre da amostra. Esta é uma consequência direta das reações para gerar quimiluminescência quando o $\mathrm{O}_{3}$ está presente em excesso no meio. Segundo a norma ASTM D 5504, a linearidade do método deste trabalho é maior que $10^{5}\left(10^{1}\right.$ a $10^{6} \mu \mathrm{g} \mathrm{m}^{-3}$ de enxofre). ${ }^{19}$ Por causa da alta sensibilidade do detector SCD, a concentração mínima detectável do analito é normalmente menor que a concentração do $\mathrm{O}_{3}$.

Através da otimização da resposta do detector se evita que desvios de linearidade ocorram devido à saturação do amplificador do mesmo. Para avaliar a relação entre a concentração de metanotiol e a resposta em área do SCD-DP, foram selecionados os ensaios com tubos de permeação e com mistura gasosa pressurizada, analisados com atenuação 1 e 100 respectivamente, cujos dados se encontram resumidos na Tabela 4. Nesta tabela a área esperada é calculada assumindo resposta linear, enquanto que a área observada reflete a resposta do sistema cromatográfico. Uma razão maior que $90 \%$ entre as áreas, esperada e observada, confirma a relação linear.

Para a elaboração do diagrama de dispersão foram usadas as concentrações, correspondentes às áreas obtidas, calculadas pelo fator de correção considerado quando o SCD-DP trabalha com atenuação 100. O fator de correlação do diagrama foi de 0,9900 , o que confirma a relação linear entre a concentração do metanotiol e sua resposta em área.

Para os compostos monossulfurados, exceto $\mathrm{SO}_{2}$, a faixa de trabalho considerada neste estudo foi de 30 a $800.000 \mu \mathrm{g} \mathrm{m}^{-3}$; em função dos MRC disponíveis. Assim, a resposta do SCD-DP é linear acima de 4 ordens de magnitude. Deve-se registrar que a referência teórica é de $10^{5}$ para a faixa de trabalho relacionada a enxofre, enquanto que a linearidade determinada em teste prático foi de $10^{4}$ para faixa de trabalho relacionada a composto sulfurado.

Na calibração da resposta de $\mathrm{SO}_{2}$ usando mistura gasosa pressurizada (Tabela 5) a área média obtida para a concentração de $498 \pm$ $6 \mathrm{mg} \mathrm{m}^{-3}$ apresentou-se consideravelmente menor que a área média para a concentração de $109 \pm 3 \mathrm{mg} \mathrm{m}^{-3}$. Por esse motivo, a linearidade para $\mathrm{SO}_{2}$ está comprometida a partir da concentração de $109 \mathrm{mg}$ $\mathrm{m}^{-3}$. Assim sendo, neste estudo a faixa de trabalho considerada para detecção de $\mathrm{SO}_{2}$ é de 0,1 a $100 \mathrm{mg} \mathrm{m}^{-3}$.

\section{Identificação e quantificação de compostos sulfurados em diversas correntes de refinaria}

A combinação das três técnicas usadas no reconhecimento de sulfurados permitiu a identificação de 18 compostos sulfurados presentes nas diversas amostras. Exceto o COS, os demais compostos sulfurados eluem em ordem crescente de seus pontos de ebulição. Alguns compostos sulfurados nas amostras não foram

Tabela 4. Verificação de linearidade do MESH na faixa de trabalho de 5 a $60 \mathrm{mg} \mathrm{m}^{-3}$

\begin{tabular}{lccccc}
\hline Tipo de MRC & Número de ensaios & Concentração $\left(\mathrm{mg} \mathrm{m}^{-3}\right)$ & Área média observada & Área esperada & Razão área observada/esperada \\
\hline mistura gasosa & 8 & $63,02 \pm 5,99$ & 10712199 & 10630365 & 1,00770 \\
tubo permeação & 10 & $8,19 \pm 0,27$ & 1566300 & 1542056 & 1,01572 \\
tubo permeação & 4 & $4,95 \pm 0,25$ & 953448 & 1005012 & 0,94869 \\
\hline
\end{tabular}

Tabela 5. Verificação de linearidade do $\mathrm{SO}_{2}$ na faixa de trabalho de 4 a $100 \mathrm{mg} \mathrm{m}^{-3}$

\begin{tabular}{lccccc}
\hline Tipo de MRC & Número de ensaios & Concentração $\left(\mathrm{mg} \mathrm{m}^{-3}\right)$ & Área observada & Área esperada & Razão área observada/esperada \\
\hline mistura gasosa & 5 & $102 \pm 14$ & 14269320 & 14198064 & 1,00502 \\
tubo permeação & 2 & $5,42 \pm 0,38$ & 754999,04 & 754446,15 & 1,00073 \\
tubo permeação & 8 & $6,96 \pm 0,56$ & 969754,12 & 968809,07 & 1,00098 \\
tubo permeação & 5 & $4,07 \pm 0,27$ & 566845,20 & 566530,59 & 1,00056 \\
tubo permeação & 7 & $4,15 \pm 0,61$ & 577666,33 & 577666,33 & 1,00000 \\
tubo permeação & 6 & $4,72 \pm 0,71$ & 657620,99 & 657008,45 & 1,00093 \\
\hline
\end{tabular}


identificados; porém, as reações de enxofre quimiluminescente induzido por $\mathrm{O}_{3}$ possuem espectro idêntico para as diferentes espécies com mesmo número de átomos de enxofre. Desse modo, os compostos não identificados são determinados com precisão igual a aqueles identificados.

\section{Corrente de gás residual da URE}

$\mathrm{O}$ cromatograma da Figura 3 mostra os sulfurados presentes no gás residual da URE antes do envio à incineração (flare). Cinco compostos sulfurados foram identificados: $\mathrm{H}_{2} \mathrm{~S}, \mathrm{COS}, \mathrm{SO}_{2}$, metanotiol (MESH) e metiletilsulfeto (MES). Cabe assinalar que os compostos COS, MESH e MES não são detectados na análise cromatográfica para avaliação de $\mathrm{H}_{2} \mathrm{~S}$ e $\mathrm{SO}_{2}$ por meio de detector de condutividade térmica (DCT).

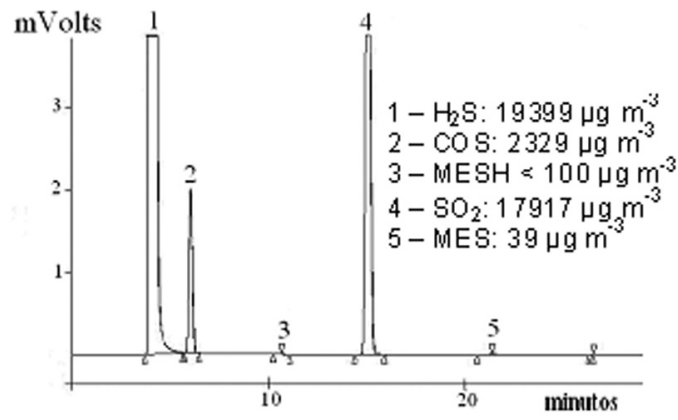

Figura 3. Cromatograma da análise por SCD-DP (sistema Varian-Sievers) do gás residual da URE: $\mathrm{H}_{2} \mathrm{~S}, \mathrm{COS}, \mathrm{MESH}, \mathrm{SO}_{2}$ e MES

\section{Gases tratados com DEA}

Neste caso os teores de $\mathrm{H}_{2} \mathrm{~S}$ podem variar de 25.000 a valores menores que $1.000 \mathrm{mg} \mathrm{m}^{-3}$. Quando o teor de $\mathrm{H}_{2} \mathrm{~S}$ é menor que $1000 \mathrm{mg} \mathrm{m}^{-3}$ é possível realizar o monitoramento utilizando a pesquisa de sulfurados por SCD. A Figura 4 ilustra um cromatograma típico dessa corrente gasosa, e os dados de quantificação estão na Tabela 6 .

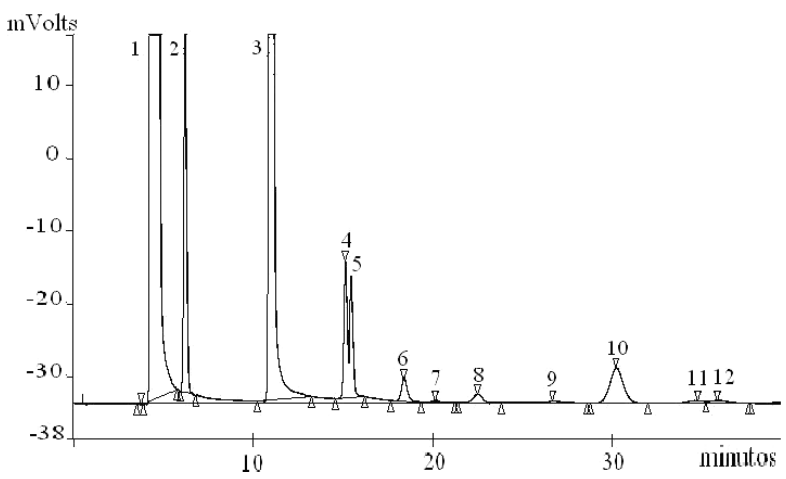

Figura 4. Cromatograma da análise por SCD-DP (sistema Varian-Sievers) do gás da FCC tratado com DEA. A identificação e a concentração dos compostos sulfurados estão na Tabela 6

\section{Correntes diversas}

Dois perfis cromatográficos obtidos de correntes de processos da refinaria estão ilustrados nas Figuras 5 e 6. Os gases do processo de destilação de petróleo para planta de combustíveis (PC) apresentaram diversidade e teores de sulfurados (Tabela 6) sistematicamente inferiores ao processo similar para planta de lubrificantes (PL), devido à diferença entre os petróleos de origem empregados em cada processo. ${ }^{21}$

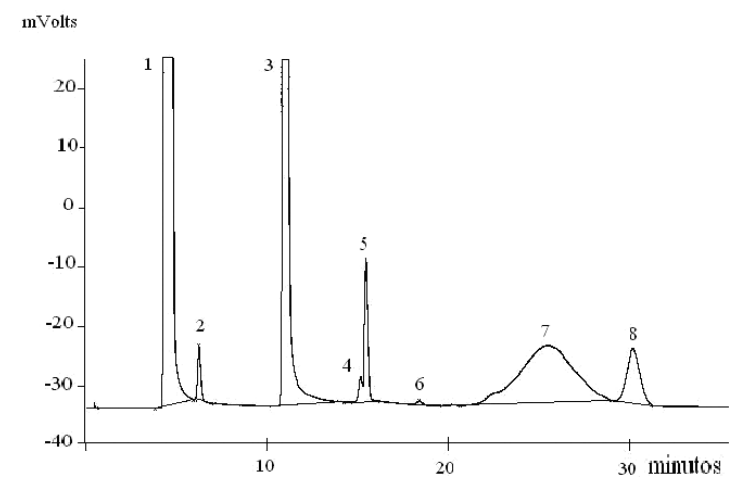

Figura 5. Cromatograma da análise por SCD-DP de gás combustível da destilação de petróleo na planta de combustíveis. A identificação e a concentração dos compostos sulfurados estão na Tabela 6

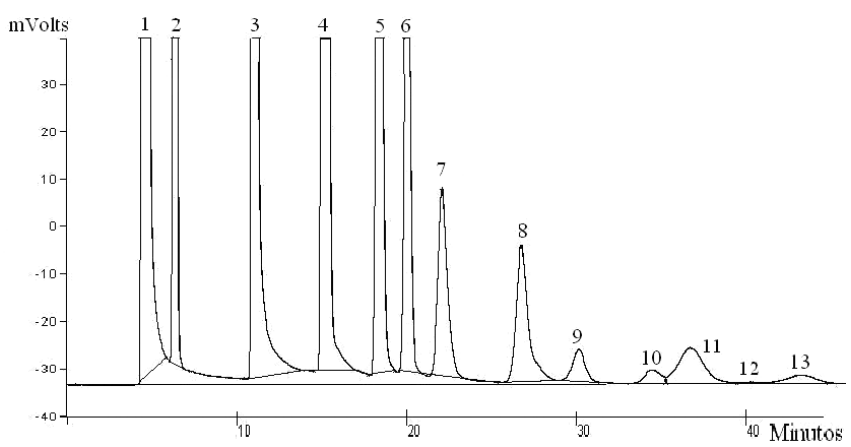

Figura 6. Cromatograma da análise por $S C D-D P$ de gás combustível da destilação de petróleo na planta de lubrificantes. A identificação e a concentração dos compostos sulfurados estão na Tabela 6

Tabela 6. Concentração de compostos sulfurados em diversas correntes gasosas de refinaria (média de 3 amostragens - valores expressos em $\mathrm{mg} \mathrm{m}^{-3}$ )

\begin{tabular}{llll}
\hline Composto & Figura 4 & Figura 5 & Figura 6 \\
\hline $\mathrm{H}_{2} \mathrm{~S}$ & 118 (pico 1) & 486 (pico 1) & 950 (pico 1) \\
$\mathrm{COS}$ & 95 (pico 2) & 1 (pico 2) & 93 (pico 2) \\
metanotiol (MESH) & 109 (pico 3) & 71,8 (pico 3) & 319 (pico 3) \\
etanotiol (ETSH) & 27 (pico 4) & & 322 (pico 4) \\
dimetilsulfeto (DMS) & 26 (pico 5) & 7,1 (picos 4 + 5) não detectado \\
2-propanotiol & 15 (pico 6) & 0,3 (pico 6) & 164 (pico 5) \\
1-propanotiol & 13 (pico 7) & não detectado & 80,3 (pico 6) \\
2-(2-metil)propanotiol & 15 (pico 8) & não detectado & 32,5 (pico 7) \\
2-butanotiol & 13 (pico 9) & 46,5 (pico 7) & 60,5 (pico 8) \\
dimetildissulfeto (DMDS) & 24 (pico 10) & 27,3 (pico 8) & 20,3 (pico 9) \\
metiletildissulfeto (MEDS) & 13 (pico 11) & não detectado & 10,9 (pico 10) \\
dietildissulfeto (DEDS) & 13 (pico 12) & não detectado & 71,6 (pico 11) \\
2-pentanotiol & não detectado & não detectado & 0,4 (pico 12) \\
1-pentanotiol & não detectado & não detectado & 9,2 (pico 13) \\
\hline
\end{tabular}

*Coelui juntamente com o $\mathrm{SO}_{2}$.

\section{CONCLUSÕES}

O método CG-SCD-DP para a determinação de compostos sulfurados em correntes gasosas possui as seguintes características, as quais seguem protocolo analítico similar ao publicado no método ASTM D 5504:19 limites de quantificação na ordem de $13 \mu \mathrm{g} \mathrm{m}^{-3}$ para monossulfurados, $70 \mu \mathrm{g} \mathrm{m}^{-3}$ para dissulfurados e $100 \mu \mathrm{g} \mathrm{m}^{-3}$ para $\mathrm{SO}_{2}$ - relacionados à otimização do plasma e reação de ozonização com vácuo eficiente; determinação de faixa de trabalho para 
$\mathrm{SO}_{2}\left(0,1 \mathrm{a} 100 \mathrm{mg} \mathrm{m}^{-3}\right)$ - relacionada à otimização do material de amostragem e das linhas de transferência para sistema de injeção no CG; equimolaridade - critério para determinação específica de $\mathrm{H}_{2} \mathrm{~S}$, COS, tióis e sulfetos pois existem diferenças que, em nível de $\mu \mathrm{g} \mathrm{m}^{-3}$, podem não serem desejadas. Para o $\mathrm{SO}_{2}$ ficou evidente a necessidade de calibração especial em todos os ensaios com amostras de refinaria.

As cartas cromatográficas disponibilizadas neste trabalho e os teores estimados de compostos sulfurados proporcionam dados que podem ser usados na avaliação do desempenho dos processos envolvidos, bem como do próprio controle do processo Claus na URE. As cartas cromatográficas constituem um guia para ser usado em novos ensaios de determinação do teor de sulfurados nestas mesmas correntes para avaliar as condições atuais de cada processo de redução dos teores de enxofre.

\section{MATERIAL SUPLEMENTAR}

O material suplementar, disponível gratuitamente na forma de arquivo .PDF em http://www.quimicanova/sbq.org.br, apresenta os seguintes dados: conjunto completo de reações que ocorrem em uma URE (Tabela 1S); informações sobre os tubos de permeação usados na calibração e na identificação de compostos sulfurados (Tabela 2S); lista de densidades e de pontos de ebulição de diversos compostos sulfurados (Tabela 3S); sumário de FC obtidos na calibração do SCDDP usando tubos de permeação (Tabela 4S) e, composição típica dos gases da URE da REDUC (Tabela 5S).

\section{AGRADECIMENTOS}

A M. C. F. Schmidt (REDUC/Petrobras) por sua importante colaboração através da coleta de dados dos experimentos realizados neste trabalho.

\section{REFERÊNCIAS}

1. The U.S. Environmental Protection Agency, Point Sources Committee, Emission Inventory Improvement Program; Emission Factors for Petroleum Industry, 1995, vol. 1, chap. 5.

2. Rocha, J. C.; Rosa, A. H.; Cardoso, A. A.; Introdução à Química Ambiental, Bookman: Porto Alegre, 2004, cap. 1 e 3.

3. El-Bishtawi, R.; Haimour, N.; Fuel Proc. Technol. 2004, 86, 245.

4. Zarenezhard, B.; J. Ind. Eng. Chem. 2009, 15, 143.

5. Sabback, O. A.; Environ. Int. 1993, 19, 41.

6. Braga, B.; Español, I.; Conejo, J.; Barros, M.; Spencer, M.; Porto, M.; Nucci, N.; Juliano, N.; Eiger, S.; Introdução à Engenharia Ambiental, Prentice Hall: São Paulo, 2002, cap. 10.
7. The U.S. Environmental Protection Agency, Office of Enforcement and Compliance Assurance; Profile of the Petroleum Refining Industry, 1995.

8. The U.S. Environmental Protection Agency, Office of Solid Waste; Sulfur Recorery: Background Report, 1998.

9. Mariano, J. B.; Impactos Ambientais do Refino do Petróleo, Interciência: Rio de Janeiro, 2005, cap. 2 e 3.

10. Shreve, R. N.; Brink Jr., J. A.; Indústrias de Processos Químicos, $4^{\mathrm{a}}$ ed., Guanabara Koogan: Rio de Janeiro, 1997. cap. 7, 19 e 37.

11. The U.S. Environmental Protection Agency, Point Sources Committee, Emission Inventory Improvement Program; Preferred and Alternative Methods for Estimating Air Emissions from Oil and Gas Field Production and Processing Operations, 1999, vol. 2, chap. 10.

12. van Nisselroy, F. M. T.; Laga, J. A.; Catal. Today 1993, 16, 263.

13. Yan, X.; J. Chromatogr., A 1999, 842, 267.

14. Yan, X..; J. Chromatogr., A 2002, 976, 3.

15. Gras, R. L.; Luong, J. C.; Mustacich, R. V.; Shearer, R. L.; J. ASTM Int. $\mathbf{2 0 0 5}, 2,7$.

16. Gras, R.; Luong, J.; Carter, V.; Sieben, L.; Cortes, H.; J. Chromatogr., A 2009, 1216, 2776.

17. Navas, M.; Jiménez, A. M.; Crit. Rev. Anal. Chem. 2000, 30, 153.

18. Pereira, K. S.; Dissertação de Mestrado, Universidade Federal do Rio de Janeiro, Brasil, 2006.

19. American Society for Testing and Materials; Standard Test Method for Determination of Sulfur Compounds in Natural Gas and Gaseous Fuels by Gas Chromatography and Chemiluminescence, norma ASTM D 5504, West Conshohocken, 2008.

20. Miller, K. E.; Thomas, J. B.; J. Chromatogr, A 2003, 1007, 117.

21. Meites, L.; Handbook of Chemical Engineering Calculations, McGrawHill: New York, 2004.

22. American Society for Testing and Materials; Standard Practice for Calibration Techniques Using Permeation Tubes, norma ASTM D 3609, West Conshohocken, 2005.

23. American Society for Testing and Materials; Standard Guide for Intercomparing Permeation Tubes to Establish Traceability, norma ASTM D 4298, West Conshohocken, 2008.

24. American Society for Testing and Materials; Standard Practice for Testing Flame Ionization Detectors Used in Gas or Supercritical Fluid Chromatography, norma ASTM E 594, West Conshohocken, 2006.

25. Chapman, R. L.; Calibration of Stack Gas Instrumentation, ASTM International: New York, 1976.

26. Cohn, P. E.; Analisadores Industriais, Interciência: Rio de Janeiro, 2006.

27. Collins, C. H.; Braga, G. L.; Bonato, P. S.; Introdução a Métodos Cromatográficos, $7^{\mathrm{a}}$ ed., Ed. da UNICAMP: Campinas, 1997. 
Júlio Carlos Afonso*

Departamento de Química Analítica, Instituto de Química, Universidade Federal do Rio de Janeiro, Av. Athos da Silveira Ramos, 149, 21941-909 Rio de Janeiro - RJ, Brasil

Kátia da Silva Pereira\#

Petróleo Brasileiro S. A., Refinaria Duque de Caxias, Av. Washington Luis, km 113,7, 25213-005 Duque de Caxias - RJ, Brasil

Tabela 1S. Conjunto completo de reações que ocorrem em uma URE ${ }^{3,12}$

\begin{tabular}{|c|c|c|}
\hline Estágio & Reações & Comentários \\
\hline \multirow{10}{*}{$\begin{array}{l}\text { Conversão térmica } \\
\text { na câmara de } \\
\text { combustão }\end{array}$} & $\mathrm{H}_{2} \mathrm{~S} \rightarrow \mathrm{H}_{2}+1 / 2 \mathrm{~S}_{2}$ & Decomposição direta \\
\hline & $2 \mathrm{H}_{2} \mathrm{~S}+\mathrm{O}_{2} \rightarrow \mathrm{S}_{2}+2 \mathrm{H}_{2} \mathrm{O}$ & Oxidação \\
\hline & $2 \mathrm{H}_{2} \mathrm{~S}+3 \mathrm{O}_{2} \rightarrow 2 \mathrm{SO}_{2}+2 \mathrm{H}_{2} \mathrm{O}$ & Oxidação \\
\hline & $2 \mathrm{H}_{2} \mathrm{~S}+\mathrm{SO}_{2} \rightarrow 3 / 2 \mathrm{~S}_{2}+2 \mathrm{H}_{2} \mathrm{O}$ & Reação de Claus \\
\hline & $\mathrm{S}_{2} \rightarrow 1 /{ }_{3} \mathrm{~S}_{6} \rightarrow 1 / 4 \mathrm{~S}_{8}$ & Condensação \\
\hline & $\mathrm{CH}_{4}+3 / 2 \mathrm{O}_{2} \rightarrow \mathrm{CO}+2 \mathrm{H}_{2} \mathrm{O}$ & Oxidação \\
\hline & $\mathrm{CO}+1 / 2 \mathrm{~S}_{2} \rightarrow \mathrm{COS}$ & Geração de compostos \\
\hline & $\mathrm{CH}_{4}+2 \mathrm{~S}_{2} \rightarrow \mathrm{CS}_{2}+2 \mathrm{H}_{2} \mathrm{~S}$ & sulfurados \\
\hline & $\mathrm{CH}_{4}+2 \mathrm{O}_{2} \rightarrow \mathrm{CO}_{2}+2 \mathrm{H}_{2} \mathrm{O}$ & Oxidação \\
\hline & $\mathrm{NH}_{3}+3 / 2 \mathrm{O}_{2} \rightarrow \mathrm{N}_{2}+2 \mathrm{H}_{2} \mathrm{O}$ & Decomposição \\
\hline \multirow{4}{*}{$\begin{array}{l}\text { Conversão } \\
\text { catalítica } \\
\text { nos reatores }\end{array}$} & $2 \mathrm{H}_{2} \mathrm{~S}+\mathrm{SO}_{2} \rightarrow 3 / 2 \mathrm{~S}_{2}+2 \mathrm{H}_{2} \mathrm{O}$ & Reação de Claus \\
\hline & $\mathrm{S}_{2} \rightarrow 1 /{ }_{3} \mathrm{~S}_{6} \rightarrow 1 / 4 \mathrm{~S}_{8}$ & Condensação \\
\hline & $\mathrm{COS}+\mathrm{H}_{2} \mathrm{O} \rightarrow \mathrm{CO}_{2}+\mathrm{H}_{2} \mathrm{~S}$ & Hidrólise \\
\hline & $\mathrm{CS}_{2}+2 \mathrm{H}_{2} \mathrm{O} \rightarrow \mathrm{CO}_{2}+2 \mathrm{H}_{2} \mathrm{~S}$ & Hidrólise \\
\hline Resfriamento & $\mathrm{S}_{2} \rightarrow 1 /{ }_{3} \mathrm{~S}_{6} \rightarrow 1 / 4 \mathrm{~S}_{8}$ & Condensação \\
\hline
\end{tabular}

Tabela 2S. Informações sobre os tubos de permeação usados na calibração e na identificação dos compostos sulfurados

\begin{tabular}{|c|c|c|c|c|}
\hline Composto sulfurado & Geometria do tubo & Tipo de tubo & Identificação do certificado & Taxa de permeação \\
\hline \multirow[t]{2}{*}{ Sulfeto de hidrogênio $\left(\mathrm{H}_{2} \mathrm{~S}\right)$} & Comprimento: $1,4 \mathrm{~cm}$ & Tubo de vida útil extendida & $10-16251$ & $682 \mathrm{ng} \mathrm{min}^{-1} \pm 2 \%$ a $30^{\circ} \mathrm{C}$ \\
\hline & $90 \mathrm{~F} 3$ & Tubo de massa em gel & F-22303 & $28,5 \mathrm{ng} \mathrm{min}^{-1} \pm 5 \%$ a $40{ }^{\circ} \mathrm{C}$ \\
\hline \multirow[t]{2}{*}{ Sulfeto de carbonila (COS) } & $30 \mathrm{~F} 3$ & Tubo de massa em gel & F-22240 & $641 \mathrm{ng} \mathrm{min}-1 \pm 5 \%$ a $30^{\circ} \mathrm{C}$ \\
\hline & $90 \mathrm{~F} 3$ & Tubo de massa em gel & F-18851 & $227 \mathrm{ng} \mathrm{min}^{-1} \pm 5 \%$ a $40^{\circ} \mathrm{C}$ \\
\hline \multirow[t]{2}{*}{ Metanotiol $\left(\mathrm{CH}_{3} \mathrm{SH}\right)$} & Comprimento: $2,3 \mathrm{~cm}$ & Tubo de alta emissão & $89-15082$ & $1113 \mathrm{ng} \mathrm{min}^{-1} \pm 2 \%$ a $30{ }^{\circ} \mathrm{C}$ \\
\hline & Comprimento: $4,0 \mathrm{~cm}$ & Tubo de emissão padrão & $33-22934$ & $335 \mathrm{ng} \min ^{-1} \pm 2 \%$ a $40^{\circ} \mathrm{C}$ \\
\hline \multirow[t]{2}{*}{ Dióxido de enxofre $\left(\mathrm{SO}_{2}\right)$} & Comprimento: $3,9 \mathrm{~cm}$ & Tubo de baixa emissão & $56-16134$ & $1293 \mathrm{ng} \mathrm{min}^{-1} \pm 2 \%$ a $30^{\circ} \mathrm{C}$ \\
\hline & $50 \mathrm{~T} 3$ & Tubo de massa em gel & T-21945 & $192 \mathrm{ng} \min ^{-1} \pm 5 \%$ a $40{ }^{\circ} \mathrm{C}$ \\
\hline Dimetilsulfeto $\left(\left(\mathrm{CH}_{3}\right)_{2} \mathrm{~S}\right)$ & Comprimento: $4,5 \mathrm{~cm}$ & Tubo padrão de emissão & $19-22243$ & $214 \mathrm{ng} \min ^{-1} \pm 2 \%$ a $30^{\circ} \mathrm{C}$ \\
\hline Dimetildissulfeto $\left(\left(\mathrm{CH}_{3}\right)_{2} \mathrm{~S}_{2}\right)$ & Comprimento: $2,0 \mathrm{~cm}$ & Tubo de alta emissão & $89-22935$ & $469 \mathrm{ng} \mathrm{min}^{-1} \pm 5 \%$ a $40^{\circ} \mathrm{C}$ \\
\hline
\end{tabular}

Fonte: Certificados dos tubos de permeação MRC, fornecidos por VICI Metronics. Tubo de vida útil extendida = Extended life tube; Tubo de massa em gel = wafer device; Tubo de alta emissão = High emission; Tubo de emissão padrão = Standard device ; Tubo de baixa emissão = Low emission . (Os termos em inglês são informados nos certificados dos tubos de permeação).

*e-mail: julio@iq.ufrj.br

"Atualmente no Centro de Pesquisas Leopoldo Miguez de Mello (CENPES/PETROBRAS) 
Tabela 3S. Lista de densidades e de pontos de ebulição de diversos compostos sulfurados ${ }^{19-21}$

\begin{tabular}{lcc}
\hline Pico e composto sulfurado & Densidade & Ponto de ebulição, ${ }^{\circ} \mathrm{C}$ \\
\hline 1- sulfeto de hidrogênio & 1,1857 (gás) & $-60,7$ \\
2- sulfeto de carbonila & 1,24 a -87 ${ }^{\circ} \mathrm{C}$ & $-49,9$ \\
3- dióxido de enxofre & 1,434 a $0{ }^{\circ} \mathrm{C}$ & $-10,0$ \\
4- metanotiol & 0,8665 a $20 / 4{ }^{\circ} \mathrm{C}$ & 6,2 \\
5- etanotiol & 0,8391 a $20 / 4{ }^{\circ} \mathrm{C}$ & 35,0 \\
6- dimetilsulfeto & 0,8483 a $20 / 4{ }^{\circ} \mathrm{C}$ & 37,3 \\
7- dissulfeto de carbono & $1,261\left(20 / 20{ }^{\circ} \mathrm{C}\right)$ & 46,5 \\
8- 2-propanotiol & 0,8143 a $20 / 4{ }^{\circ} \mathrm{C}$ & 52,6 \\
9- 2-(2-metil)propanotiol & 0,8002 a $20 / 4{ }^{\circ} \mathrm{C}$ & 65,0 \\
10- 1-propanotiol & 0,8415 a $20 / 4{ }^{\circ} \mathrm{C}$ & 67,0 \\
11- metiletilsulfeto & 0,8422 a $20 / 4{ }^{\circ} \mathrm{C}$ & 67,0 \\
12- 2-butanotiol & 0,8299 a $20 / 4{ }^{\circ} \mathrm{C}$ & 85,0 \\
13- 2-metil-1-propanotiol & 0,8343 a $20 / 4{ }^{\circ} \mathrm{C}$ & 88,7 \\
14- dimetildissulfeto & 1,0625 a $20 / 4{ }^{\circ} \mathrm{C}$ & 109,7 \\
15- dietildissulfeto & 0,9931 a $20 / 4{ }^{\circ} \mathrm{C}$ & 154,0 \\
\hline
\end{tabular}

Tabela 4S. Sumário de FC obtidos na calibração do SCD-DP usando tubos de permeação

\begin{tabular}{lccccc}
\hline $\begin{array}{l}\text { Composto } \\
\text { sulfurado }\end{array}$ & $\begin{array}{c}\text { Temp. de } \\
\text { geração }\left({ }^{\circ} \mathrm{C}\right)\end{array}$ & Replicatas & $\begin{array}{c}\text { Maior } \\
\text { valor }\end{array}$ & Mediana & $\begin{array}{c}\text { Menor } \\
\text { valor }\end{array}$ \\
\hline $\mathrm{H}_{2} \mathrm{~S}$ & 30 & 44 & 0,05 & 0,05 & 0,04 \\
$\mathrm{H}_{2} \mathrm{~S}$ & 40 & 39 & 0,05 & 0,05 & 0,04 \\
$\mathrm{COS}$ & 30 & 27 & 0,03 & 0,02 & 0,02 \\
$\mathrm{COS}$ & 40 & 63 & 0,02 & 0,02 & 0,02 \\
$\mathrm{MESH}$ & 30 & 44 & 0,04 & 0,04 & 0,03 \\
$\mathrm{MESH}$ & 40 & 62 & 0,05 & 0,04 & 0,04 \\
$\mathrm{SO}_{2}$ & 30 & 28 & 0,04 & 0,04 & 0,03 \\
$\mathrm{DMS}$ & 30 & 40 & 0,04 & 0,03 & 0,03 \\
DMDS & 40 & 22 & 0,06 & 0,05 & 0,03 \\
\hline
\end{tabular}

Tabela 5S. Composição típica (\% vol) dos gases da URE da REDUC, obtida por cromatografia gasosa com detector de condutividade térmica (DCT)

\begin{tabular}{lccc}
\hline Carga & $\%$ vol & Gás de saída & $\%$ vol \\
\hline Sulfeto de hidrogênio & 75,85 & Sulfeto de hidrogênio & 1,55 \\
Dióxido de carbono & 23,23 & Dióxido de carbono & 18,47 \\
Oxigênio & 0,16 & Oxigênio & 0,45 \\
Nitrogênio & 0,32 & Nitrogênio & 79,02 \\
Metano & 0,01 & Dióxido de enxofre & 0,46 \\
Etano & 0,02 & Sulfeto de carbonila & 0,05 \\
Eteno & 0,04 & - & - \\
Propano & 0,37 & - & - \\
\hline
\end{tabular}

